\section{Ameloblastoma sólido plexiforme multiquístico en cuerpo mandibular izquierdo. Reporte de caso}

\section{Solid plexiform multicystic ameloblastoma in left mandibular body. Case report}

\section{Resumen}

En el 2017 la Organización Mundial de la Salud describió al ameloblastoma como una neoplasia benigna, localmente invasiva, consistente en una proliferación de epitelio odontogénico, que usualmente presenta un patrón folicular o plexiforme, y se encuentra dentro de un estroma fibroso. Cursa con un lento crecimiento y gran afinidad por el tejido óseo, presenta un alto índice de recurrencia, aproximadamente de 60 a $80 \%$, si no es removido de la manera adecuada. Algunas variantes como la uniquística y la desmoplásica cuentan con un mejor pronóstico que la sólida o multiquística. Representan el $1 \%$ de todos los tumores de la mandíbula y el $11 \%$ de todos los tumores odontogénicos. Se presenta el caso clínico de un paciente masculino de 43 años de edad que acude a nuestro servicio en el 2016 por presentar aumento de volumen con un año de evolución, asintomático, y con principios de movilidad dental en la zona mandibular izquierda. A la exploración intraoral se aprecia aumento de volumen en región mandibular con un incremento de expansión de las corticales, lo cual es involucrado desde la pieza dentaria 36 a la 41, no presenta sintomatología dolorosa a la palpación ni a la percusión.

Palabras clave: Ameloblastoma; Reconstrucción mandibular; Neoplasias de la boca; Patología.

\begin{abstract}
In 2017, World Health Organization described the ameloblastoma like a benign, locally invasive neoplasm, that consists in proliferation of odontogenic epithelium, which usually presents a folicular or plexiform pattern, and it is found inside a fibrous stroma. It presents slow development and affinity with woven osseus tissue, with high recurrence $(60 \%-80 \%)$ if it is not well resected. Variants like uniquistic and desmoplastic have better prognostic than solid o multicyst. It represents the $1 \%$ of tumors of the jaw and the $11 \%$ of odontogenic tumors. The following case is a male patient, 43 years old which goes to our service in 2016 by increasing volume of a year of evolution, asymptomatic, and with principles of tooth mobility in left side of the jaw. Intraoral examination shows increase in volume in mandibular region with increased cortical expansion which involves teeth 36 to 41, asymptomatic to palpation or percussion of teeth involved.
\end{abstract}

Keywords: Ameloblastoma; Mandibular reconstruction; Mouth Neoplasms; Pathology.

\section{Caso Clínico}

Mauricio González Rebattú ${ }^{1, a}$, Ana María Nieto Munguía 1,a, Carlos Muñoz Torres ${ }^{1, b}$, Adalberto Sánchez Sánchez ${ }^{1, \mathrm{~b}}$

1 Servicio Cirugía Oral y Maxilofacial. Hospital Regional $1^{\circ}$ de Octubre. D.F. México, México.

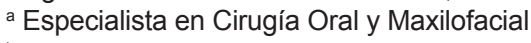

${ }^{\mathrm{b}}$ Cirujano Dentista

Correspondencia:

Adalberto Sánchez Sánchez

Correo electrónico:

adalbertosanchezsanchez@gmail.com Av. Instituto Politécnico Nacional Núm. 1669, Col. Magdalena de las Salinas, Del. Gustavo A. Madero, México, D.F.

\section{Coautores: \\ Mauricio Gonzales Rebattú \\ mauriciorebattu@yahoo.com.mx \\ Ana María Nieto Munguía \\ annie_tit@hotmail.com \\ Carlos Muñoz Torres \\ enriquemt3@gmail.com}

Conflicto de intereses: Los autores declaran no tener conflictos de interés.

Fuente de financiamiento: Autofinanciado

Fecha de recepción: 07/04/18

Fecha de aceptación: 03/07/18

( ) Los autores. Este artículo es publicado por la revista Odontología Sanmarquina de la Facultad de Odontología, Universidad Nacional Mayor de San Marcos. Este es un artículo de acceso abierto, distribuido bajo los términos de la licencia Creative Commons Atribucion - No Comercia_Compartir Igual 4.0 Internacional. (http://creativecommons.org/licenses/by-nc-sa/4.0/) que permite el uso no comercial, distribución y reproducción en cualquier medio, siempre que la obra original sea debidamente citada. 


\section{Introducción}

El ameloblastoma es de origen odontogénico y fue descrito por primera vez por Cuzack en 1827. Posteriormente en 1885 Malassez propuso el nombre de "adamantinoma" debido a la formación de una masa dura parecida al hueso, motivo por el cual Churchill sugirió el cambio por ameloblastoma en $1934^{1}$.

El ameloblastoma es el segundo tumor odontogénico más común después del odontoma, representa el 11\% de todos los tumores odontogénicos. Es localmente agresivo pero histológicamente benigno, presente un origen epitelial y puede ser originado a partir del esmalte, folículo dental o del ligamento periodontal ${ }^{2}$.

De acuerdo con la clasificación de la OMS en el 2017 se puede clasificar en sólido o multiquístico, extraóseo o periférico, y uniquístico ${ }^{3}$. Histopatológicamente se clasifica como folicular, plexiforme, acantomatoso, granular, desmoplásico y basaloide. Radiográficamente puede ser unilocular o multilocular. Estos aparecen como lesiones radiolúcidas de tamaño variable.

El ameloblastoma usualmente afecta a los adultos jóvenes de la cuarta a quinta década de la vida sin distinción de sexo. Clínicamente inicia con un crecimiento lento, asintomático, y en estadios más avanzados con pérdida de piezas dentarias, maloclusión, parestesia, dificultad para la apertura bucal y asimetría facial ${ }^{4}$. Radiográficamente se aprecia como una lesión radiolúcida multilocular en forma de pompas de jabón o panal de abejas. En el Ameloblastoma de tipo desmoplásico se aprecia un patrón mixto radiolúcido y radiopaco, el cual da la impresión de una lesión fibroósea. Su recurrencia con tratamiento conservador es de $90,9 \%$, mientas que el tratamiento radical tan solo de $9,1 \%{ }^{3}$. Aproximadamente el $80 \%$ de los ameloblastomas se encuentran en la mandíbula, con predilección en la región posterior seguido por la parte anterior en la mandíbula y en la zona posterior del maxilar. El patrón desmoplásico tiene predilección hacia la región anterior del maxilar. Raramente se llegan a encontrar en el seno maxilar.

El problema de la recurrencia inicia al momento de realizar el diagnóstico. Primero se debe identificar, según la biopsia, el tipo de ameloblastoma por la histología, y así determinar si el tratamiento será conservador o radical. Varios estudios han reportado una alta recurrencia posterior al tratamiento conservador comparado con el tratamiento radical ${ }^{5-9}$.

\section{Reporte del caso}

Paciente masculino de 43 ańos es referido en el 2016 al servicio de Cirugía Maxilofacial del Hospital Regional $1^{\circ}$ de Octubre, México. Presentó aumento de volumen con un año de evolución, asintomático y con principios de movilidad dental en la zona anterior y lado izquierdo de la mandíbula, no presentó algún tipo de enfermedad crónico-degenerativa (Figura 1). A la exploración intraoral se aprecia aumento de volumen en región mandibular con incremento de expansión de corticales involucrando las piezas dentarias 36 a 41, sin dolor a la palpación ni a la percusión (Figura 2).

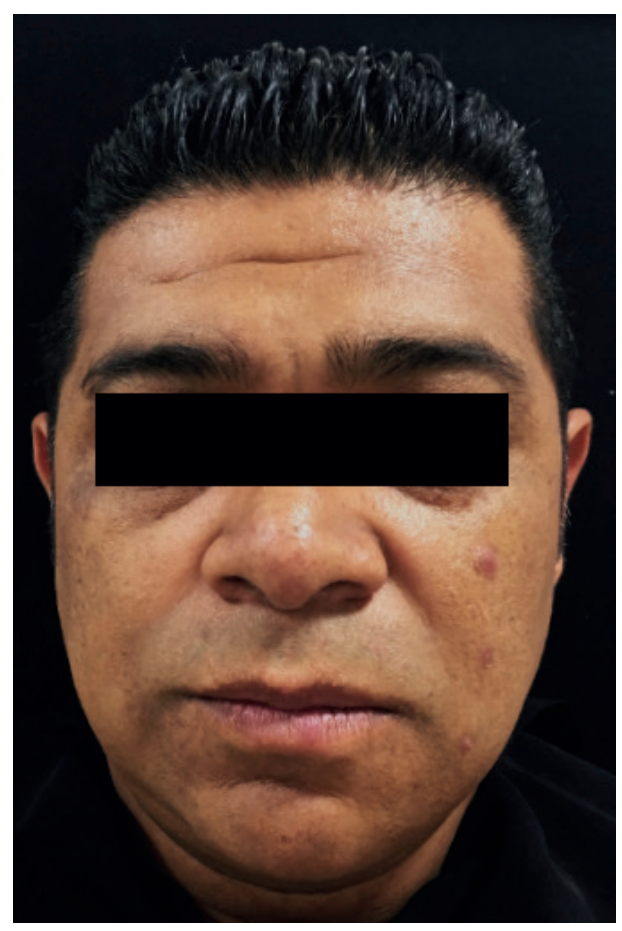

Figura 1. Fotografía frontal del paciente, sin mostrar aumentos de volumen extraoral

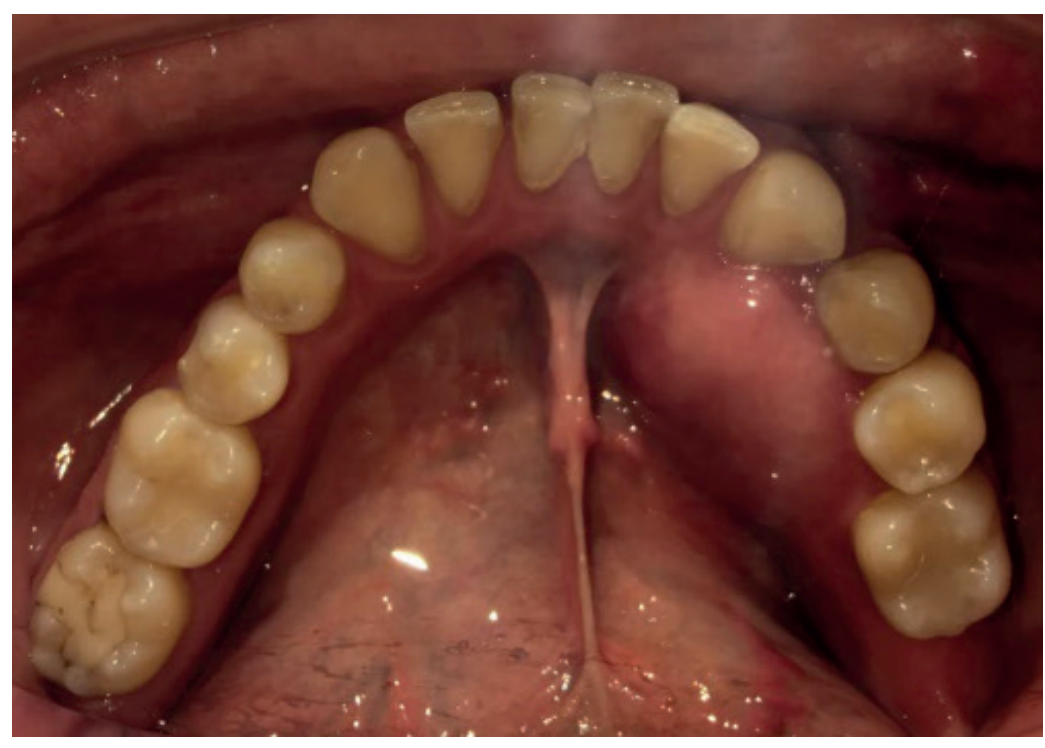

Figura 2. Fotografía intraoral inicial del paciente donde se observa un aumento de volumen en cortical lingual de órganos dentarios del 32 al 36 
En la ortopantomografia se observa una zona radiolúcida, multiloculada, con aspecto de pompas de jabón de bordes bien definidos, localizada desde la pieza dentaria 37 a la 42 (Figura 3) motivo por el cual se decide tomar biopsia incisional en la misma cita.

El estudio histopatológico identifica una neoplasia de naturaleza odontogénica formada por una capsula de tejido fibroso laxo bien vascularizado y parcialmente revestida por un epitelio de aspecto ameloblástico, con una capa basal cuboidal a columnar, sobre la que se encuentran varias capas de células parecidas al retículo estrellado del órgano del esmalte. La capa basal muestra numerosas yemas de la que se desprenden islas y múl- tiples cavidades quísticas revestidas por epitelio ameloblástico similar al del revestimiento principal sobre un estroma de tejido fibroso maduro. Hacia la periferia con presencia de trabéculas de hueso lamelar vital. Dando como impresión diagnostica resultado compatible con "ameloblastoma solido plexiforme multiquístico". Motivo por el cual se inicia el protocolo para realizar resección mandibular en bloque. Posteriormente se solicita tomografía del macizo facial, en la cual realizamos reconstrucción volumétrica observando el grado de compromiso de la lesión (Figura 4), se realizó la medición en el punto de mayor longitud en un corte axial obteniendo $26 \mathrm{~mm} \times 43 \mathrm{~mm}$ (Figura 5).

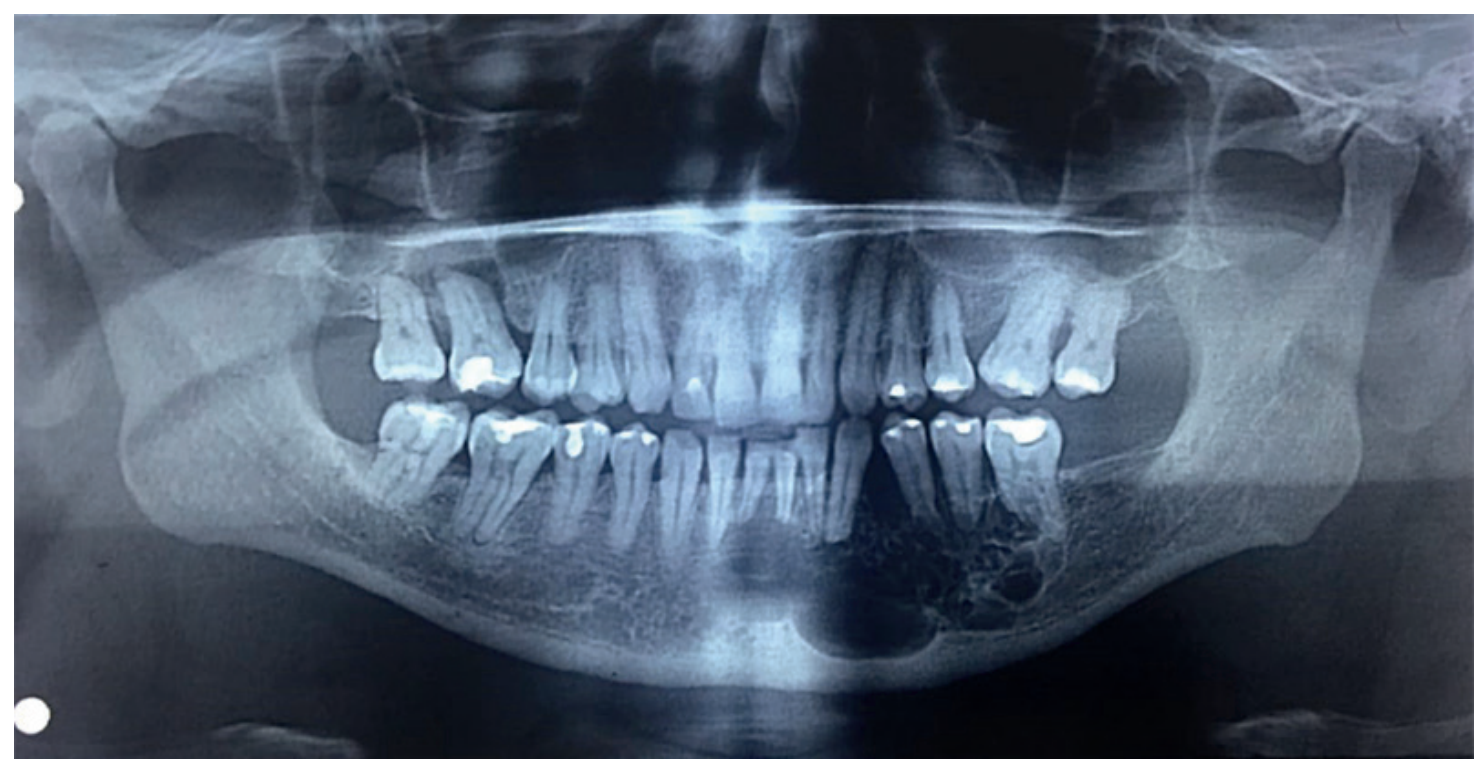

Figura 3.Ortopantomografía donde se aprecia zona radiolúcida multilobulada ubicada en cuerpo mandibular de lado izquierdo, que involucra de OD 36 a OD 42

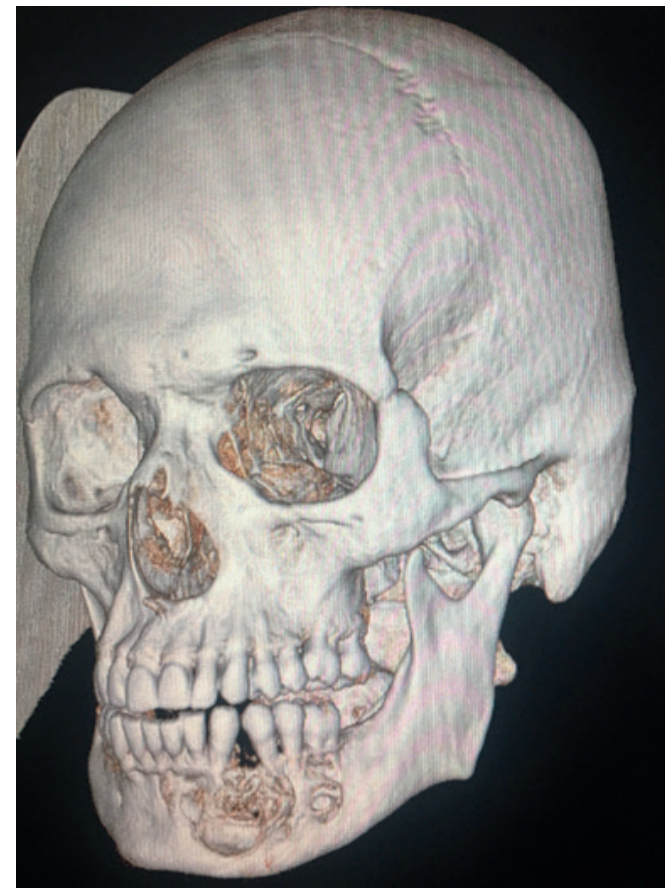

Figura 4. Reconstrucción tridimensional de macizo facial

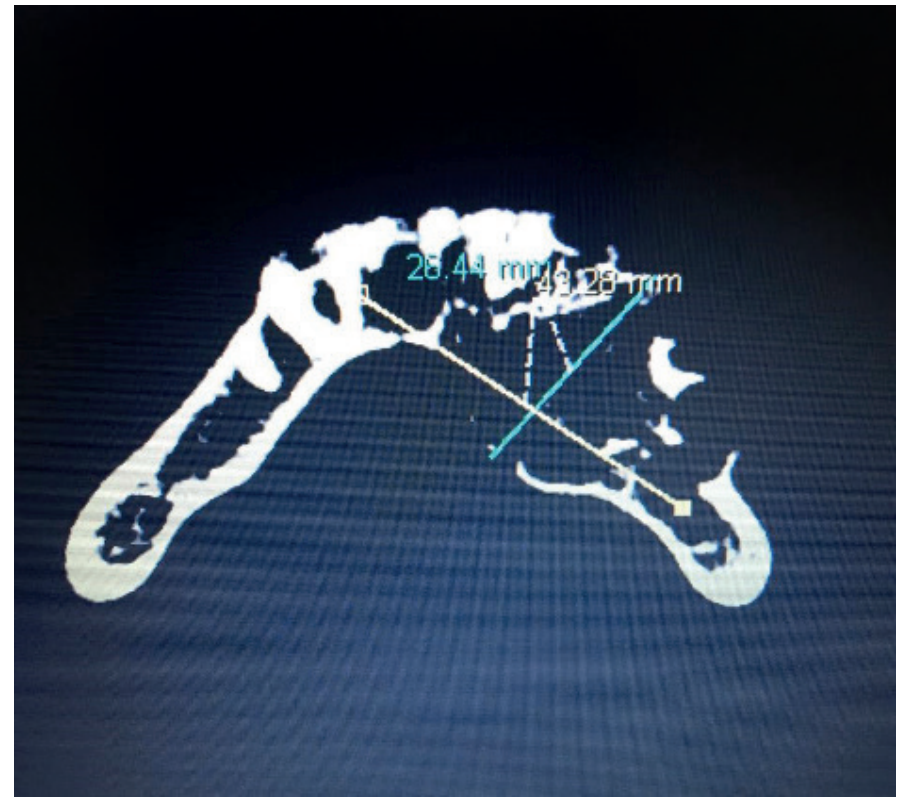

Figura 5. TC corte axial donde se podemos medir el tamaño de la lesión 
El paciente es ingresado al quirófano para realizarle la resección mandibular con bordes de seguridad de la lesión y colocándosele una placa de reconstrucción mandibular sistema 2.4, posterior a ello, se cierra por planos y se envía la pieza quirúrgica a estudio histopatológico (Figuras 6 y 7 ).

Actualmente el paciente se encuentra con una adecuada evolución postoperatoria sin manifestar un rechazo biológico del material de reconstrucción, con revisiones semanales por la consulta externa del servicio de cirugía maxilofacial, sin datos de recidiva de lesión (Figuras 8 A y B, y 9).

Se solicita la ortopantomografía y tomografía de control postoperatorio donde se observa placa de reconstrucción en adecuada posición (Figuras 10 A y B).

\section{Discusión}

El comportamiento del ameloblastoma se describe como un tumor benigno de crecimiento lento, localmente agresivo, y que presenta una tendencia a la invasión, a la destrucción ósea y a la recurrencia si no se retira de la manera más adecuada.

El ameloblastoma plexiforme es una variante del ameloblastoma, el cual tiene características clínicas, radiológicas e histológicas específicas. Se localiza con mayor frecuencia en la región del cuerpo mandibular y representa el $86 \%$ del total de los ameloblastomas. Existe controversia respecto a su origen, ya que se ha citado que proviene del epitelio reducido del esmalte y del epitelio de revestimiento de algunos quistes de origen odontogénico.

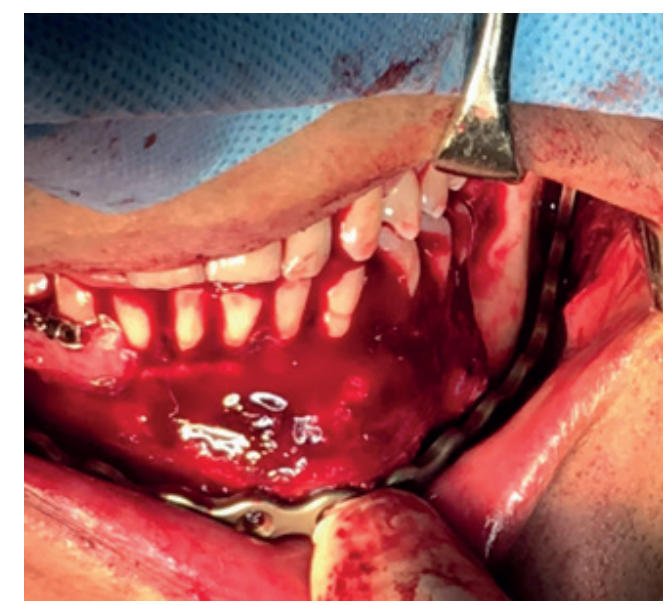

Figura 6. Colocación de placa de reconstrucción mandibular

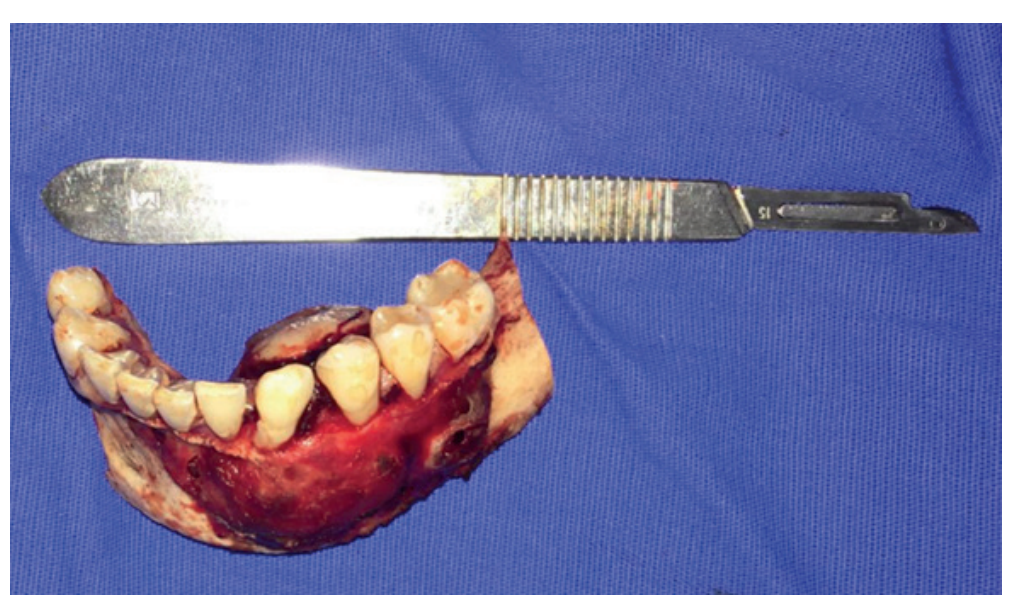

Figura 7. Pieza quirúrgica de cuerpo y sínfisis mandibular

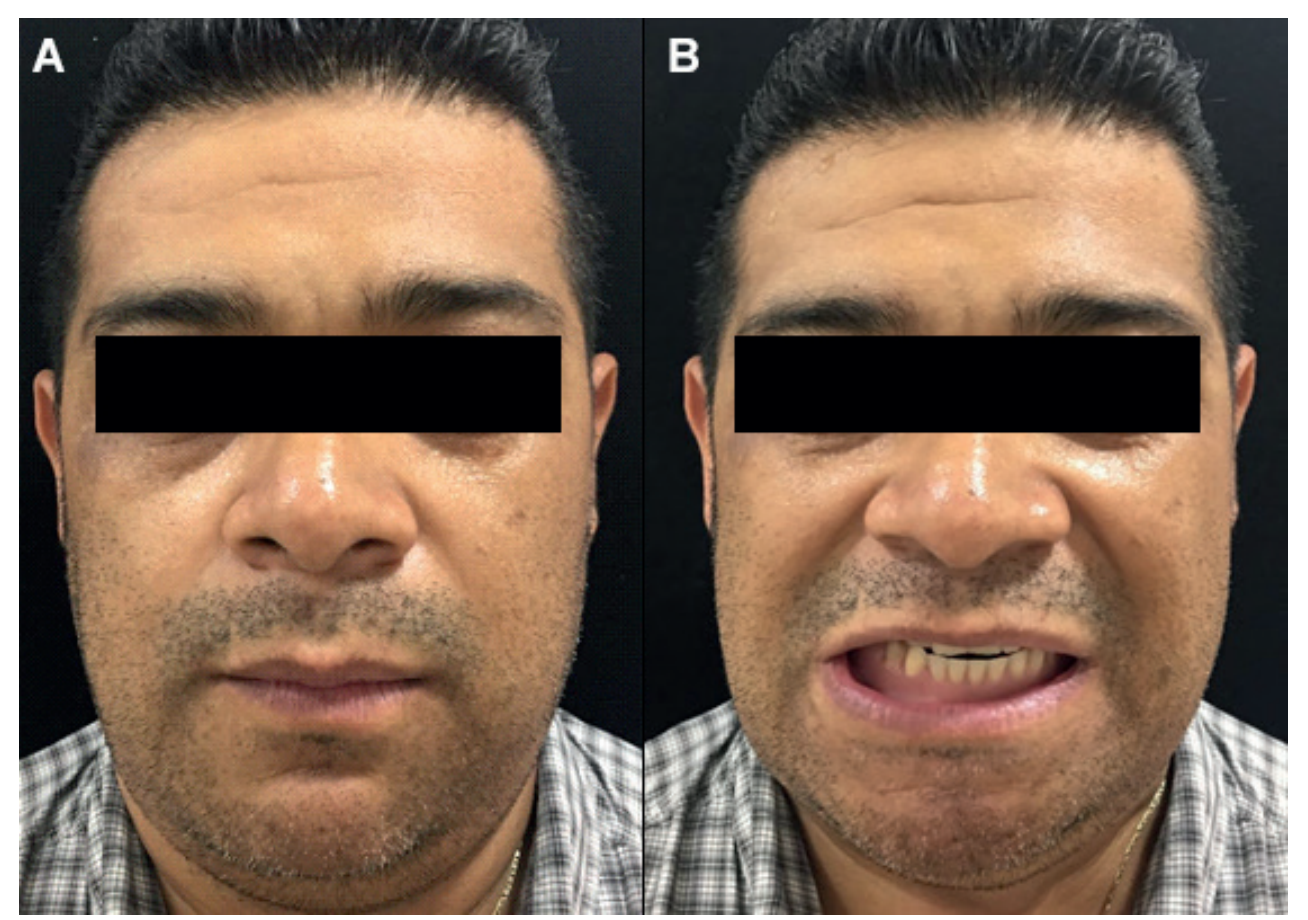

Figura 8 A y B. Postoperatorio a los 6 meses donde se aprecia una adecuada recuperación, con rehabilitación intraoral mediante prótesis parcial removible 


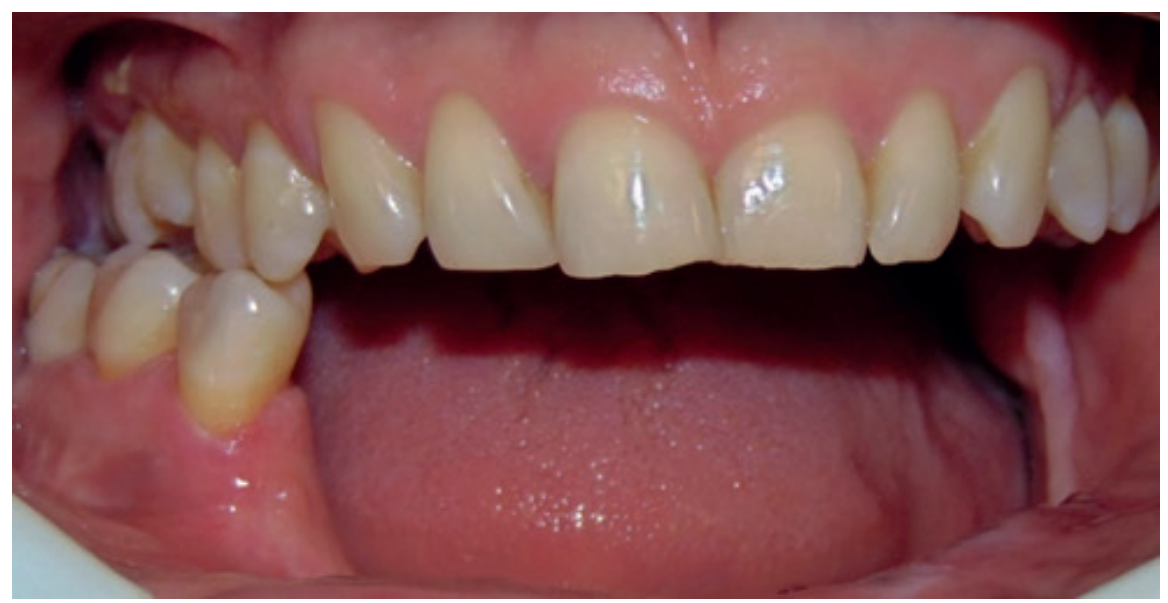

Figura 9.Postoperatorio intraoral a los 6 meses donde se aprecia adecuado proceso de cicatrización

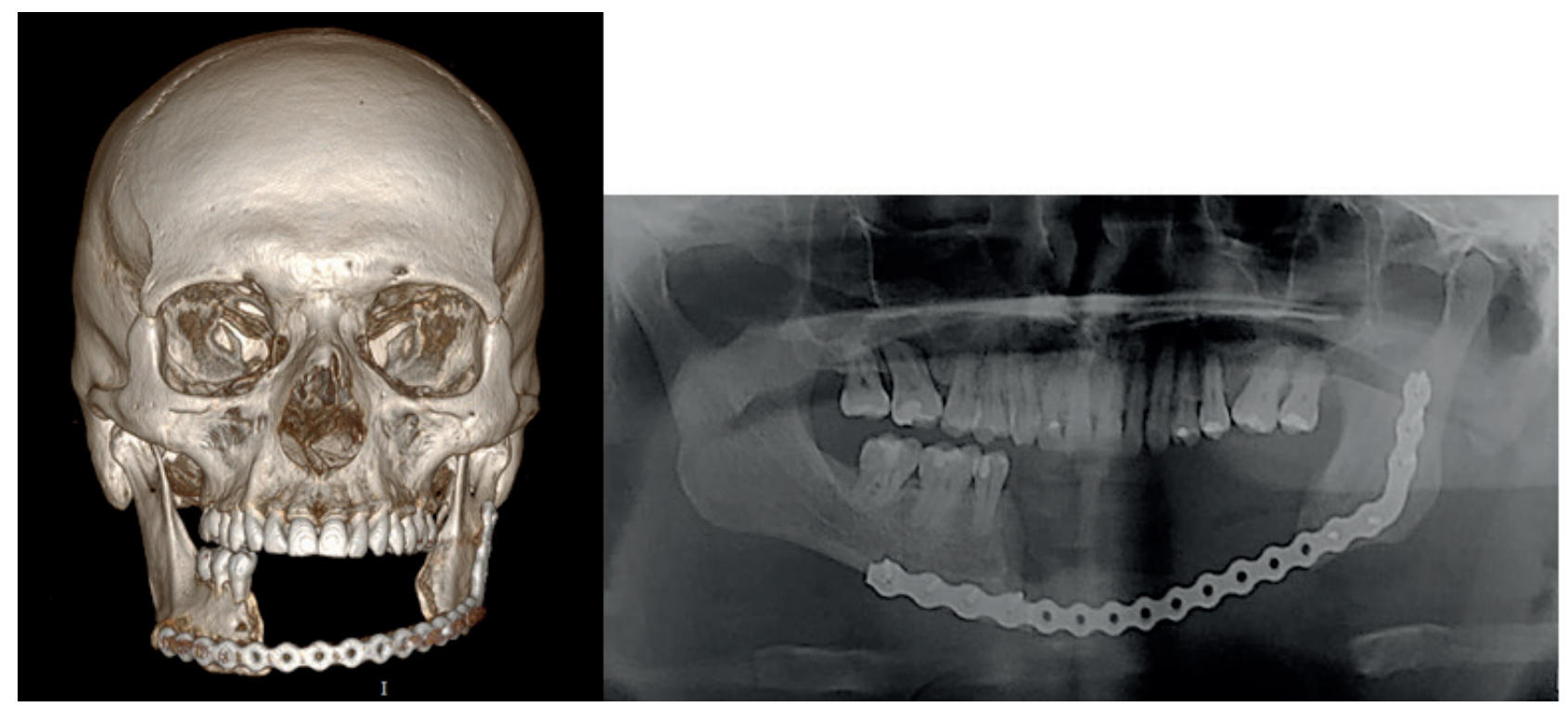

Figura 10 A y B. Tomografía y ortopantomografía postoperatorio donde se aprecia material de reconstrucción en adecuada posición y función

Se considera que el ameloblastoma plexiforme tiene un comportamiento agresivo, con tendencia a infiltrar de forma irregular al tejido óseo, por lo cual en base a la literatura se realizó una resección en bloque mandibular con márgenes de seguridad de $10 \mathrm{~mm}$, así como un planteamiento para un injerto óseo libre en un segundo tiempo quirúrgico a expensas de la alta recidiva de esta patología 5,6.

Este plan de tratamiento fue elegido debido a la alta recurrencia de la lesión y a su alta tasa de recidiva cuando es tratada de manera conservadora $(90 \%)$ mientras que de manera radical la recidiva es de solo el $9 \%$.

Olaitan et al. ${ }^{5}$ en su estudio llevado a cabo en 1993 encontraron una recurrencia de $40 \%$ al tratamiento conservador y una recurrencia de $8,3 \%$ al tratamiento radical.

El tratamiento radical es la primera elección en el tratamiento para las formas folicular y no uniquísticas, Carlson et al. ${ }^{7}$ recomiendan márgenes de seguridad de $1-1,5 \mathrm{~cm}$. Cuando la resección es prevista para un tumor de grandes dimensiones, inmediatamente debe de ser considerada una reconstrucción con un injerto libre inmediato ${ }^{8}$. Vayvada et al. ${ }^{9}$ en su estudio con $11 \mathrm{pa}-$ cientes reportaron que no hay recurrencia posterior a la reconstrucción con injerto libre en un seguimiento por 29,3 meses. Chaine et al. ${ }^{10}$ reportaron resultados similares en su estudio en 44 pacientes en un seguimiento por 53 meses.

Actualmente en el hospital Pitié-Salpêtrière se ha propuesto un algoritmo de tratamiento, donde se recomienda un tratamiento conservador en lesiones menores de $5 \mathrm{~cm}$ sin invasión periférica y tratamiento radical en el resto de lesiones ${ }^{9}$. El seguimiento se realiza cada 6 meses por 5 años mediante estudios radiográficos y clínicos.

Sachs recomienda tratar los ameloblastomas mediante escisión quirúrgica y osteotomía periférica de 2 a $3 \mathrm{~mm}$ ${ }^{10}$. Pogrel reporta resultados tratando los ameloblastomas con curetaje o resección junto a una crioterapia con nitrógeno líquido. Describe que el uso de nitrógeno líquido posterior a la enucleación de los ameloblastomas disminuye las tasas de recidivas ${ }^{11}$. 
El ameloblastoma al ser una entidad patológica benigna pero localmente agresiva, y la cual suele pasar desapercibida hasta estadios más avanzados, debe de mantenerse bajo vigilancia estrecha por al menos 5 años. En nuestro caso clínico se optó por colocación de placa de reconstrucción mandibular y no en un injerto óseo libre debido a la alta recurrencia que presenta este tipo de ameloblastoma, y el cual se tiene programado en caso de no existir recidiva. Se deben de realizar estudios radiográficos al menos cada 6 meses para descartar recidiva.

Actualmente el paciente cuenta con 18 meses de seguimiento, donde no se observan datos clínicos ni radiográficos de recidiva de lesión, se envió a servicio de prótesis maxilofacial donde se realizó rehabilitación mediante prótesis removible mucodentosoportada.

Se concluye que para realizar una resección en bloque, esta nos permite la remoción total de la lesión con una baja tasa de recurrencia, aunque dentro de las desventajas se encuentra la deformidad facial y el compromiso de la función. Existen múltiples alternativas para la reconstrucción del bloque reseccionado, la literatura reporta que en defectos menores a $5 \mathrm{~cm}$ se pueden utilizar los injertos libres y mayores a esta cifra lo ideal es realizar un injerto microvascularizado de peroné, con la finalidad de evitar defectos estéticos y pensar en una posterior rehabilitación con implantes endoóseos. Actualmente se continuará con seguimiento radiológico hasta cumplir 24 meses de postoperatorio y en caso de no mostrar evidencia de recurrencia, se iniciará un protocolo para utilizar una reconstrucción con un injerto microvascularizado de peroné y la colocación de implantes dentales.

\section{Referencias bibliográficas}

1. Martínez RB, Elizondo LX, Mosqueda TA, Aguirre UJ. Ameloblastoma desmoplásico con predominio quístico: Presentación de un caso clínico. Rev Esp Cirug Oral y Maxilofac. 2016;38(3):155-158. Doi: https://doi. org/10.1016/j.maxilo.2014.03.005

2. Chukwuneke FN, Anyanechi CE, Akpeh JO, Chukwuka A, Ekwueme O.C. Clinical characteristics and presentation of ameloblastomas: an 8-year retrospective study of 240 cases in Eastern Nigeria. Br J Oral Max- illofac Surg. 2016 May; 54(4):384-7. Doi: 10.1016/j. bjoms.2015.08.264

3. Laborde A, Nicot R, Wojick T, Ferri J, Raoul G. Améloblastome des maxillaires : prise en charge thérapeutique et taux de récidive. Annales françaises d'Oto-rhino-laryngologie et de Pathologie Cervico-faciale. 2017; 1341(1):6-10.

4. Adel K.E, Chan J.K., Grandis J.R., Takata T., Slootweg P.J..World Health Organization Classification Head and Neck Tumours. Lyon: IARC Press; 2017. p. 215-218.

5. Olaitan AA, Adeola DS, Adekeye EO. Ameloblastoma: clinical features and management of 315 cases from Kaduna. Nigeria. J Craniomaxillofac Surg 1993; 21(4):351355. Doi: org/10.1016/S1010-5182(05)80497-4

6. Hertog D, van der Waal I. Ameloblastoma of the jaws: a critical reappraisal based on a 40-years single institution experience. Oral Oncol 2010;46(3):61-64. Doi: 10.1016/j.oraloncology.2009.11.002

7. Carlson ER, Marx RE. The ameloblastoma: primary, curative surgical management. J Oral Maxillofac Surg 2006;64(2):484-494. Doi: 10.1016/j.joms.2005.11.032

8. Vayvada H, Mola F, Menderes A, Yilmaz M. Surgical management of ameloblastoma in the mandible: segmental mandibulectomy and immediate reconstruction with free fibula or deep circumflex iliac artery flap (evaluationof the long-term esthetic and functional results). J Oral Maxillofac Surg 2006; 64(3):1532-1539. Doi: 10.1016/j.joms.2005.11.065

9. Chaine A, Pitak-Arnnop P, Dhanuthai K, Ruhin-Poncet B, Bertrand JC, Bertolus C. A treatment algorithm for managing giant mandibular ameloblastoma: 5-year experiences in a Paris university hospital. Eur J Surg Oncol 2009; 35(4):999-1005. Doi: 10.1016/j. ejso.2009.04.006

10. Sachs S. Surgical excision with peripheral ostectomy: a definitive, yet conservative, approach to the surgical management of ameloblastoma. J Oral Maxillofac Surg 2006; 64(5):476-483. Doi: 10.1016/j.joms.2005.12.001

11. Pogrel MA, Sampson DE. Management of mandibular ameloblastoma: the clinical basis for a treatment algorithm. J Oral Maxillofac Surg. 1999; 57(2):1074-7. Doi: 10.1016/S0278-2391(99)90328-2 